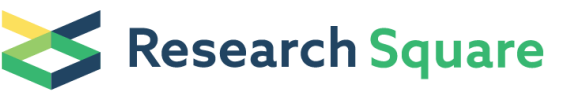 \\ Preprints are preliminary reports that have not undergone peer review. \\ They should not be considered conclusive, used to inform clinical practice, or referenced by the media as validated information.
}

\section{Nitrogen Fixation by Paenibacillus Polymyxa WLY78 is Responsible for Cucumber Growth Promotion}

\section{Shuang Liu}

China Agricultural University

\section{Qin Li}

China Agricultural University

\section{Yongbin Li}

China Agricultural University

\section{Tianyi Hao}

China Agricultural University

\section{Sanfeng Chen ( $\nabla$ chensf@cau.edu.cn )}

China Agrigulture University https://orcid.org/0000-0003-2956-9025

\section{Research Article}

Keywords: Plant growth-promoting bacteria, Paenibacillus polymyxa WLY78, cucumber, nitrogen fixation, $15 \mathrm{~N}$ isotope dilution

Posted Date: August 17th, 2021

DOl: https://doi.org/10.21203/rs.3.rs-758683/v1

License: (c) (i) This work is licensed under a Creative Commons Attribution 4.0 International License. Read Full License

Version of Record: A version of this preprint was published at Plant and Soil on January 31 st, 2022. See the published version at https://doi.org/10.1007/s11104-022-05307-6. 


\section{Abstract}

Aims To study nitrogen contribution to cucumber derived from nitrogen fixation of Paenibacillus polymyxa WLY78.

Methods The nif gene cluster deletion mutant ( $\triangle$ nifB- $V)$ of $P$. polymyxa WLY78 is constructed by a homologous recombination method. The GFP-labeled $\triangle n i f B-V$ mutant was used to inoculate cucumber and to study colonization by confocal laser scanning microscope. The effects of plant-growth promotion were investigated by greenhouse experiments. The nitrogen fixation contribution was estimated by ${ }^{15} \mathrm{~N}$ isotope dilution experiments.

Results Deletion of nif gene cluster of P. polymyxa WLY78 resulted in complete loss of nitrogenase activity. Observation by laser confocal microscopy revealed $\Delta$ nifB- $V$ mutant can effectively colonize cucumber root, stem and leaf tissues, like wild-type P. polymyxa WLY78. Greenhouse experiments showed that inoculation with P. polymyxa WLY78 can significantly enhance the lengths and dry weights of cucumber roots and shoots, but inoculation with $\triangle$ nifB-V mutant can not. ${ }^{15} \mathrm{~N}$ isotope dilution experiments showed that cucumber plants derive $25.93 \%$ nitrogen from nitrogen fixation performed by $P$. polymyxa WLY78, but the $\Delta$ nifB- $V$ mutant nearly can not provide nitrogen for plant growth.

Conclusions This present study demonstrates that nitrogen fixation performed by P. polymyxa WLY78 is responsible for cucumber growth promotion.

\section{Introduction}

Nitrogen is one of the most important nutrients in plant growth, but plants can not directly use nitrogen in the atmosphere. Biological nitrogen fixation (BNF) is a process in which nitrogen-fixing microorganisms reduce nitrogen in the air to ammonia through nitrogenases. Biological nitrogen fixation is an important part of the natural nitrogen cycle (Dart 1986) and plays an important role in the sustainable development of agriculture (Raymond et al. 2004). Nitrogen-fixing microorganisms include symbiotic nitrogen-fixing bacteria, free-living nitrogen-fixing bacteria and associative nitrogen-fixing bacteria (Xu et al. 2017). Associative nitrogen-fixing bacteria can colonize root surface cells, invade plant roots, and form close contact with plants, thereby promote plant growth (Baldani et al. 1997). Associative nitrogen-fixing bacteria promote the absorption of nitrogen by non-legume plants (Geddes et al. 2015). Biological nitrogen fixation not only reduce the use of nitrogen fertilizer, but also improve soil fertility and the absorption of nutrients by crops (Farrar et al. 2014).

It has been reported that the associative nitrogen-fixing bacteria play an important role in promoting growth of non-legumes by fixing nitrogen and producing phytohormone (Chalk 1991). In earlier research, ${ }^{15} \mathrm{~N}$ isotope and $\mathrm{N}$ balance studies have shown that several sugarcane varieties obtain over $60 \%$ of their nitrogen ( $>150 \mathrm{~kg} \mathrm{~N}^{-1}$ year $^{-1}$ ) from biological nitrogen fixation performed by diazotrophs (Boddey et al. 1995). Diazotrophic bacteria present in the mucilage of aerial roots 
contribute $29-82 \%$ of the N nutrition of Sierra Mixe maize (Van Deynze et al. 2018). Inoculation with nitrogen-fixing Klebsiella pneumoniae 342 (Kp342) increased total $\mathrm{N}$ and $\mathrm{N}$ concentration in the wheat plant (Iniguez et al. 2004). Inoculation of the rhizobacteria including Azospirillum brasilense and Azospirillum lipoferum contributed up to $20-50 \%$ of the total nitrogen requirement of the oil palm seedlings through nitrogen fixation (Amir et al. 2003). Diazotrophic Paenibacillus beijingensis BJ-18 provided nitrogen for wheat, maize and cucumber plants and promoted plant growth, nitrogen uptake and metabolism (Li et al. 2019). A recombinant nitrogen-fixing Pseudomonas protegens Pf-5 X940 was constructed by introducing the nif genes of Pseudomonas stutzeri A1501 via the X940 cosmid to the beneficial rhizobacterium Pseudomonas protegens Pf-5, and inoculation of Arabidopsis, alfalfa, tall fescue and maize with Pf-5 X940 increased the ammonium concentration in soil and plant productivity under nitrogen-deficient conditions (Fox et al. 2016; Setten et al. 2013). Inoculation with Azospirillum brasilense Ab-V5 cells enriched with exopolysaccharides and polyhydroxybutyrate enhances the productivity of maize under low $\mathrm{N}$ fertilizer input (Oliveira et al. 2017)

Paenibacillus polymyxa WLY78 is a nitrogen-fixing bacterium containing a compact nif gene cluster consisting of 9 genes (nifBHDKENXhesAnifV) (Wang et al. 2013『Xie et al. 2014). In addition to nitrogen fixation, this bacterium has the ability of phosphate solubilization and IAA production (Xie et al. 2016). Also, this bacterium can produce fusaricidins that are a class of cyclic lipopeptide antibiotics to inhibit plant pathogenic fungi (Li et al. 2019). These specific traits suggest that $P$. polymyxa WLY78 is a member of plant growth-promoting bacteria (PGPB) and has great potential as an inoculant in agriculture. However, the nitrogen contribution to plants derived from nitrogen fixation of $P$. polymyxa WLY78 is unclear. In this study, the nif gene cluster deletion mutant ( $\triangle$ nifB- $V)$ of $P$. polymyxa WLY78 is constructed. Comparisons of $P$. polymyxa wild-type and $\triangle$ nifB- $V$ mutant in colonization, plant-growth promotion and nitrogen fixation contribution rate are investigated. Our study will provide foundation for application of $P$. polymyxa WLY78 as a biofertilizer in agriculture.

\section{Materials And Methods}

Bacterial strains, plasmids, media and growth conditions

Bacterial strains and plasmids used in this study are summarized in Table 1. Paenibacillus polymyxa WLY78 was isolated from the rhizosphere of bamboo in Beijing (Wang et al. 2013). P. polymyxa WLY78 and $\triangle$ nifB- $V$ mutant were routinely grown in LB medium (per liter contains: $10 \mathrm{~g} \mathrm{NaCl}, 5 \mathrm{~g}$ yeast and $10 \mathrm{~g}$ tryptone) at $30^{\circ} \mathrm{C}$ with shaking. Nitrogen-limited medium (per liter contains: $10.4 \mathrm{~g} \mathrm{Na}_{2} \mathrm{HPO}_{4}, 3.4 \mathrm{~g}$ $\mathrm{KH}_{2} \mathrm{PO}_{4}, 26 \mathrm{mg} \mathrm{CaCl} \cdot 2 \mathrm{H}_{2} \mathrm{O}, 30 \mathrm{mg} \mathrm{MgSO}, 0.3 \mathrm{mg} \mathrm{MnSO}_{4}, 36 \mathrm{mg}$ Ferric citrate, $7.6 \mathrm{mg} \mathrm{Na} \mathrm{MoO}_{4} \cdot 2 \mathrm{H}_{2} \mathrm{O}$, $10 \mathrm{mg}$ p-aminobenzoic acid, $10 \mathrm{mg}$ biotin, $4 \mathrm{~g}$ glucose as carbon source and $0.3 \mathrm{~g}$ glutamic acid as nitrogen source) (Wang et al. 2013) was used for assay of nitrogenase activity (Wang et al. 2013).

Escherichia coli JM109 was used as routine cloning. Thermo-sensitive vector pRN5101 (Zhang et al. 2013) was used for gene disruption in P. polymyxa WLY78. The recombinant plasmid pGFP300 (Hao and Chen 2017) carrying the gfp gene was used for labeling P. polymyxa strains. When appropriate, 
antibiotics were added in the following concentrations: $100 \mu \mathrm{g} / \mathrm{ml}$ ampicillin, $12.5 \mu \mathrm{g} / \mathrm{ml}$ tetracycline and $5 \mu \mathrm{g} / \mathrm{ml}$ erythromycin for maintenance of plasmids.

Table 1 Bacterial strains and plasmids used in this study.

Strain or plasmid Genotype and/or relevant characteristics

Source or reference

\section{strains}

Paenibacillus

polymyxa

WLY78 Wild-type strain

(Wang et

al. 2013)

$\Delta n i f B-V$

nif gene cluster deletion mutant

This study

GFP-tagged $P$.

polymyxa WLY78

P. polymyxa WLY78 carrying plasmid pGFP300; Tet $^{r}$

(Hao and

Chen

2017)

GFP-

tagged $\Delta$ nifB- $V$

$\Delta$ nifB- $V$ carrying plasmid pGFP300; Tet $^{r}$

This study

E. coli

JM109

General cloning host; recA1, endA1, gyrA96, thi-1, hsdR17, supE44,

relA1, $\triangle($ lac-proAB)/F'[traD36, proAB+, laclq, lacZ $\triangle \mathrm{M} 15]$

Sangon

Biotech

Co.

\section{Plasmids}

pRN5101

Temperature-sensitive E. coli-Bacillus shuttle vector, Em ${ }^{r}$

(Zhang et

al. 2013)

pRDnifB-V

nif gene cluster deletion vector based on pRN5101

This study

pGFP300

gfp gene in PHY300PLK

(Hao and Chen

2017)

Construction of $\triangle n i f B-V$ mutant of P. polymyxa WLY78

The nitrogen-fixing gene cluster deletion mutant $\triangle$ nifB-Vof $P$. polymyxa WLY78 was constructed by a homologous recombination method. For doing this, the upstream fragment and downstream fragment flanking nif gene cluster were amplified by PCR using Phanta ${ }^{\circledR}$ Max Super-Fidelity DNA Polymerase (Vazyme Biotech Co., Ltd., Nanjing, China), respectively. Primer 1 (5' CGGCCACGATGCGTCCGGCGTAGAGGATCCGCGTGGTG 
used to amplify a $950 \mathrm{bp}$-long sequence located upstream of nifB. Primer3 (5' GAAGGAATGATAACCGAAAA

AgCGTTCCCGTC 3') and Primer 4 (5' GACTGCGCAAAAGACATAATCGATAAGCTTCCTGATAAGgCAG

ACAAGGCTC 3') were used to amplify a 1107 bp-long sequence located downstream of nifV. The two fragments were then fused with $\mathrm{BamH} \otimes /$ Hind III digested pRN5101 vector using Gibson assembly master mix (New England Biolabs), generating the recombinant plasmid pRDnifB-V. Then, the recombinant plasmid pRDnifB-V was transformed into P. polymyxa WLY78 as described by Wang et al., (2018), and the marker-free deletion mutant (the double-crossover transformant) $\Delta$ nif $B$ - $V$ was selected from the initial erythromycin resistance $(\mathrm{Em})$ transformants after several rounds of nonselective growth at $39^{\circ} \mathrm{C}$ and confirmed by PCR using the primer 5 (5' GCATAAATTGTACACGTTGA $\left.3^{\prime}\right)$ and primer 6 (5' AGGCTCATAAACACCGTATC 3').

Growth and nitrogenase activity of the wild type and mutant strains

To measure growth, $P$. polymyxa WLY78 and $\triangle$ nifB- $V$ were grown in $20 \mathrm{~mL}$ of LB media in $50 \mathrm{~mL}$ flasks shaken at $200 \mathrm{rpm}$ at $30^{\circ} \mathrm{C}$ to logarithmic growth phase. The cultures were collected by centrifugation, washed three times with sterilized water and then resuspended in sufficient nitrogen medium (nitrogenlimited medium supplemented with $100 \mathrm{mM} \mathrm{NH}_{4} \mathrm{Cl}$ ) to a start $\mathrm{OD}_{600}$ of 0.02 . Every $2 \mathrm{~h}$, the growth of $P$. polymyxa WLY78 and $\triangle$ nifB-V mutant strains were determined by absorbancy at $600 \mathrm{~nm}$.

Acetylene reduction assays were performed as described previously to measure nitrogenase activity (Wang et al. 2013). P. polymyxa WLY78 and $\triangle$ nifB-V mutant strains were grown in $50 \mathrm{~mL}$ of LB medium overnight. The cultures were collected by centrifugation, washed three times with sterilized water, and then resuspended in a nitrogen-limited medium to a final $\mathrm{OD}_{600}$ of 0.4 . Then, $4 \mathrm{~mL}$ of the culture was transferred to a $26-\mathrm{mL}$ test tube and the test tube was sealed with a rubber stopper. The headspace in the tube was then evacuated and replaced with argon gas. Then, approximately $2.2 \mathrm{~mL}$ of $\mathrm{C}_{2} \mathrm{H}_{2}(10 \%$ of the headspace volume) was injected into the test tubes. After incubating the cultures at $30^{\circ} \mathrm{C}$ for the corresponding time, a $100 \mu \mathrm{L}$ gas sample was taken out and injected into gas chromatography to quantify ethylene $\left(\mathrm{C}_{2} \mathrm{H}_{4}\right)$ production. The nitrogenase activity was expressed in $\mathrm{nmol} \mathrm{C}_{2} \mathrm{H}_{4} / \mathrm{mg}$ protein/h.

Preparation of soil, seeds and bacterial suspension

The soil (0-20 cm deep topsoil) was taken from the Shangzhuang Experimental Station of China Agricultural University. The soil was low nitrogen $\left(7.8 \mathrm{mg} \mathrm{kg}^{-1}\right)$ sandy soil. After the soil was air-dried and crushed, the debris were removed with a $2 \mathrm{~mm}$ sieve to reduce heterogeneity, and then packed into plastic pots with a diameter of $20 \mathrm{~cm}$ and a height of $14 \mathrm{~cm}$. Each pot was filled with $2 \mathrm{~kg}$ of soil to grow cucumbers. No trace elements were applied during plant growth.

Cucumber seeds ("Zhongnong 8" of Beijing Shengfeng Garden Agricultural Technology Co., Ltd.) were first disinfected with $10 \%$ sodium hypochlorite for 10 minutes, then were rinsed with sterile water three 
times, and finally were distributed in a sterile petri dish with damp filter paper at room temperature $\left(25^{\circ} \mathrm{C}\right)$ for 3-5 days in the dark.

The bacterial suspension of $P$. polymyxa WLY78 and $\triangle$ nifB-V used in inoculation was prepared as follows. P. polymyxa WLY78 and $\triangle$ nifB- $V$ were inoculated into LB liquid medium, cultured at $30^{\circ} \mathrm{C}$ and 180 rpm to logarithmic growth phase, and then harvested by centrifugation and finally suspended with physiological saline $\left(0.89 \% \mathrm{~W} / \mathrm{v} \mathrm{NaCl}\right.$ in deionized water). The cell concentration was set to $10^{8}$ cells $\mathrm{mL}^{-}$ 1 .

\section{Colonization of $P$. polymyxa and $\Delta$ nifB- $V$ on cucumber}

The recombinant plasmid pGFP300 carrying the $g f p$ gene was transferred into $\Delta$ nifB- $V$ by electrotransformation as described in P. polymyxa WLY78 (Hao and Chen 2017), yielding GFP-tagged $\triangle$ nifB- $V$ mutant. The GFP-tagged $\Delta$ nifB- $V$ mutant and GFP-tagged $P$. polymyxa WLY78 suspension were obtained as described above to final concentration at $10^{8}$ cells $\mathrm{mL}^{-1}$. The sterilized cucumber seeds were sown in sterile glass bottles containing $100 \mathrm{~mL}$ of $1 / 2 \mathrm{MS}$ semi-solid agar medium, and one seed was placed in each bottle. After dark treatment for about a week, the seeds were grown into seedlings, which were transferred to a light incubator $\left(27^{\circ} \mathrm{C}, 70 \%\right.$ humidity and $16 \mathrm{~h}$ day $/ 8 \mathrm{~h}$ night, with light at $250 \mu \mathrm{mol}$ $\mathrm{m}^{-2} \mathrm{~s}^{-1}$ ) for cultivation. The seedlings were regularly watered (tap water) to $40 \%$ relative soil moisture by weighing method every 5 days. After the seedlings grow 2-3 young leaves, inoculate the cell suspension of GFP-tagged strains at the root of cucumber. Three days later, the colonization of GFP-labeled strains in cucumber tissues were placed on a slide and visualized with an excitation wavelength of $488 \mathrm{~nm}$ with a laser confocal scanning microscope (CLSM, Olympus FluoViewTM FV1000 confocal microscope). The emitted light was collected in the 500-565 nm range for GFP, and the images were collected with FV10ASW software (03.01.02.02, Olympus Europa Holding GmbH, Hamburg Germany).

\section{Greenhouse pot experiment}

The research was conducted in the greenhouse of China Agricultural University using greenhouse potting. The experimental design was arranged by random factors, with three inoculation treatments and two nitrogen level treatments. Each treatment was repeated three times, for a total of 18 pots of cucumber plants. Nitrogen level treatment included high nitrogen and low nitrogen levels. Nitrogen fertilizer was applied in the form of ${ }^{15} \mathrm{~N}$ labeled $\left(\mathrm{NH}_{4}\right)_{2} \mathrm{SO}_{4}\left(10.16 \%{ }^{15} \mathrm{~N}\right.$ atom, Shanghai Research Institute of Chemical Industry, China). The high nitrogen level was $250 \mathrm{mg} \mathrm{N} \mathrm{kg}^{-1}$ soil, and the low nitrogen level was $83 \mathrm{mg} \mathrm{N}$ $\mathrm{kg}^{-1}$ soil. Nitrogen fertilizer was applied in three times, one-third each time, and the first time was applied as a base fertilizer, followed by 7 and 14 days after transplantation.

The inoculation treatment included three treatments: inoculation of P. polymyxa WLY78 (WT), $\Delta n i f B-V$, and equal amount of physiological saline (as a control). The germinated cucumber seeds with robust and consistent growth were picked and immersed in the bacterial suspension for 20 minutes. The seeds were immersed in physiological saline for 20 minutes as a control group, and then transplanted into plastic 
pots. Four seeds were planted in each pot, and three repetitions were set for each treatment. In the first and second weeks after planting, the P. polymyxa WLY78 and $\triangle$ nifB- $V$ bacterial suspensions were reinoculated into the roots of the plants, and the control group was added with the same amount of physiological saline. Place the flower pots under the best conditions in the greenhouse $(15 \mathrm{~h}$ light $/ 9 \mathrm{~h}$ dark cycle, $25-30 / 15-20^{\circ} \mathrm{C}$ day/night temperature and $40 \%$ day $/ 60 \%$ night humidity). The seedlings were regularly watered every 3 days until the relative humidity of the soil reached $40 \%$.

Plant sample collection

On 30th day of cucumber planting, the plants were collected by destructive sampling. The whole seedling was first uprooted, and rinsed with deionized water to remove the soil attached to the root system, then the root and shoot samples were separated, and the length of the root and shoot were measured. The root and shoot samples were killed in an oven at $105^{\circ} \mathrm{C}$ for 30 minutes, and then dried at $65^{\circ} \mathrm{C}$ until constant weight for dry weight analysis. Then the dried samples were immediately frozen in liquid nitrogen for subsequent analysis.

Contribution of nitrogen by biological nitrogen fixation

The ${ }^{15} \mathrm{~N}$ isotope dilution technique was used to quantitatively determine the contribution of inoculated bacteria to cucumber biological nitrogen fixation. The above dried sample was ground, sieved with a 1 $\mathrm{mm}$ sieve and placed in a bag, and the cucumber $\mathrm{N}$ content and ${ }^{15} \mathrm{~N}$ enrichment determination were performed by DELTA V Advantage isotope ratio mass spectrometer (Thermo Fisher Scientific, Inc., United States). The cucumber without inoculation were used as references to calculate the BNF contribution. After inoculation with P. polymyxa WLY78 and $\triangle$ nifB-V, the percentage of nitrogen from cucumber biological nitrogen fixation to the nitrogen content in cucumber (\% $\mathrm{Ndfa}$ ) was calculated by the following formula:

$\%$ Ndfa $=\left(1-\frac{\% \text { NdfF }}{\% \text { NdfNF }}\right) \times 100$

Among them, \%NdfF is the ${ }^{15} \mathrm{~N}$ enrichment of cucumber stems and leaves of inoculation treatment, and\% $\mathrm{NdfNF}$ is the ${ }^{15} \mathrm{~N}$ enrichment of cucumber stems and leaves of uninoculated treatment (control group).

Statistical Analysis

Graphs were prepared using GraphPad Prism software v. 8.0 (GraphPad Software Inc., San Diego, CA, USA). Statistical analysis was performed using SPSS software version 20 (SPSS Inc., Chicago, IL, United States). One-way analysis of variance (ANOVA) was employed to check the significant differences between treatments. Means of different treatments were compared using the least significant difference (LSD) at the 0.05 or 0.01 level of probability.

\section{Results}


The nif gene cluster deletion mutant ( $\triangle$ nifB- $V$ ) of $P$. polymyxa WLY78 leads to complete loss of nitrogenase activity

P. polymyxa WLY78 contains a compact nif gene cluster consisting of 9 genes (nifB nifH nifD nifK nifE nifN nifX hesA nifV) located within a $10.5 \mathrm{~kb}$ region. The nif gene cluster deletion mutant ( $\triangle$ nifB-V) was constructed by recombination as described in Fig. 1a. The disruption of nif gene cluster was confirmed by PCR (Fig. 1b). The $\triangle$ nifB- $V$ and the wild-type strains exhibited similar growth phenotypes on nitrogenlimited medium with ammonium as nitrogen sources (Fig. 1c). However, the $\Delta$ nifB-V mutant did not have nitrogenase activity (Fig.1d), indicating that the nif gene cluster is essentially required for nitrogen fixation under nitrogen limitation.

The colonization of cucumber by GFP-Tagged $\Delta$ nifB-V mutant

The cucumber colonization by P. polymyxa WLY78 has been previously described (Hao and Chen 2017). Here, the $\triangle$ nifB- $V$ mutant was labeled with GFP and then the GFP-labeled bacteria were used to inoculate cucumber and examine colonization. After 3 days of inoculation at the root of cucumber, the samples of the cucumber roots, stems and leaves were observed under laser confocal microscopy. The cells of GFPtagged $\triangle$ nifB- $V$ mutant not only colonized on the surface of cucumber root, but also colonized interior of root, stem and leaf (Fig. 2d-f), as observed in the wild-type P. polymyxa WLY78 (Fig. 2a-c). The presence of bacterial cells was observed in the vascular bundle of the stem and the leaves. The results indicated that $P$. polymyxa WLY78 and $\triangle n$ ifB- $V$ can colonize outside and inside of cucumber tissues.

Effects of P. polymyxa WLY78 and $\Delta$ nifB-Von the growth of cucumber

Cucumber samples were collected on the 30th day after plantation, and the length and dry weight of cucumber shoots and roots were measured to evaluate the effects of inoculation with P. polymyxa WLY78 and $\Delta$ nifB- $V$ mutant on plant growth under low and high nitrogen conditions. The non-inoculated cucumber served as a control. Compared to the non-inoculated control group, cucumber inoculated with P. polymyxa WLY78 under low nitrogen conditions showed increase of $68.18 \%$ in shoots dry weight, of $59.15 \%$ in root dry weight, of $37.61 \%$ in shoot length and of $38.52 \%$ in root length, but they showed a little increase in lengths and weights under high nitrogen conditions (Fig. 3a-d). Compared with the noninoculated control group, the cucumber inoculated with the $\triangle$ nif $B-V$ mutant showed a little increase in the dry weight and length of the shoots and roots under both low and high nitrogen conditions (Fig. 3a-d). Fig. 4 is an experimental diagram of the greenhouse cultivation. The data indicate that the diazotrophic $P$. polymyxa WLY78 can effectively promote plant growth under low nitrogen conditions and disruption of nif genes encoding nitrogenase results in almost loss of the ability of promoting plant

Nitrogen fixation in cucumber provided by P. polymyxa

To estimate the contribution of $\mathrm{BNF},{ }^{15} \mathrm{~N}$ isotope dilution technique was used to analyze the inoculated cucumber grown in soil which contains ${ }^{15} \mathrm{~N}$-labeled $\left(\mathrm{NH}_{4}\right)_{2} \mathrm{SO}_{4}$ as $\mathrm{N}$ fertilizer in greenhouse conditions (Table 2). The nitrogen derived from gaseous nitrogen (\%Ndfa) in the P. polymyxa WLY78 inoculated 
cucumber and the $\triangle$ nifB- $V$ strain inoculated cucumber under low $\mathrm{N}$ conditions is $25.93 \pm 2.32 \%$ and 2.93 $\pm 6.57 \%$, respectively. Whereas, the nitrogen derived from gaseous nitrogen $(\% \mathrm{Ndfa})$ in the $P$. polymyxa WLY78 inoculated cucumber and the $\triangle$ nifB- $V$ strain inoculated cucumber under high $\mathrm{N}$ conditions is 1.54 $\pm 0.66 \%$ and $-0.22 \pm 1.02 \%$, respectively. These results indicate that the cucumber plant has incorporated the nitrogen provided by BNF of P. polymyxa WLY78 under low N conditions and BNF is inhibited by high concentration of available nitrogen in the environment. The $\triangle$ nif $B-V$ strain-inoculated cucumbers nearly did not derive nitrogen from $B N F$, consistent with the $\triangle$ nifB- $V$ strain has no nitrogenase. The data also indicate that $P$. polymyxa WLY78 can be used to provide nitrogen nutrition to plants and reduce the use of nitrogen fertilizers.

Table 2 Quantification of biological nitrogen fixation in the inoculated cucumber plants grown in soil containing high $\mathrm{N}$ and low $\mathrm{N}$ conditions.

\begin{tabular}{lll} 
Treatment & \multicolumn{2}{l}{$\%$ Ndfa } \\
\cline { 2 - 3 } & Low nitrogen & High nitrogen \\
\hline Control & - & - \\
\hline P.polymyxa WLY78 & $25.93 \pm 2.32^{\mathrm{a}}$ & $1.54 \pm 0.66^{\mathrm{a}}$ \\
\hline$\Delta$ nifB-V & $2.93 \pm 6.57^{\mathrm{b}}$ & $-0.22 \pm 1.02^{\mathrm{a}}$
\end{tabular}

The results came from three biological replicates, the error represents SD, lowercase letters $a$ and $b$ indicate that there is a significant difference between the groups $(P<0.05)$, while the same letter indicates that there is no significant difference.

\section{Discussion}

In this study, both GFP-tagged P. polymyxa WLY78 and GFP-tagged $\triangle$ nifB-V mutant are able to colonize the roots, stems, and leaves of cucumber. This result shows that the deletion of the nif gene cluster did not affect the colonization. Similarly, both of wild-type Klebsiella pneumoniae 342 and the nifH mutant can colonize wheat (Iniguez et al. 2004) and both wild-type Pseudomonas stutzeri A1501 and its nifH mutant can colonize maize root (Ke et al. 2019). Wild-type Acetobacter diazotrophicus PAl5 and the nifD mutant have the same ability of colonization in sugarcane (Sevilla et al. 2001). A difference between our study with other's is that the $\triangle$ nifB- $V$ mutant of $P$. polymyxa WLY78 has a deletion of a compact nif gene cluster comprising 9 genes (nifBHDKENXhesAnifV) and the nifH mutant or nifK mutant or nifD mutant has a deletion of a single nif gene.

The effects of $P$. polymyxa WLY78 and $\triangle n$ if $B$ - $V$ on cucumber growth under different nitrogen concentrations were further studied through greenhouse cultivation experiments. Compared to the uninoculated control and the inoculation with the $\triangle$ nifB-V mutant, inoculation with P. polymyxa WLY78 significantly increased the dry weights and lengths of cucumber shoots and roots under low nitrogen 
conditions, but this effect was not found in the cucumbers inoculated with the $\Delta$ nifB-V mutant. These results have revealed that the nitrogen fixation of $P$. polymyxa WLY78 plays an important role in promoting plant growth. Phosphate solubilization and IAA production of P. polymyxa WLY78 may exhibit a minor role in promoting plant growth. Similarly, inoculation with Pseudomonas stutzeri A1501 strain can increase the root and shoot dry weight of maize, but this effect is not found in the maize inoculated with nifH-mutant (Ke et al. 2019). In $\mathrm{N}_{2}$-deficient conditions, Kallar grass inoculated with Azoarcus sp. $\mathrm{BH} 72$ grew better and accumulated more nitrogen than plants inoculated with the nifK $\mathrm{K}$ mutant strain (Hurek, et al. 2002.). Inoculation with K. pneumoniae 342 resulted in increased dry weight, chlorophyll content, total $\mathrm{N}$, and $\mathrm{N}$ concentration of wheat in comparison with the uninoculated and nifH mutantinoculated controls (Iniguez et al. 2004).

The ${ }^{15} \mathrm{~N}$ isotope dilution technique is commonly used to determine the contribution of nitrogen-fixing bacteria to plant nitrogen. In this study, the ${ }^{15} \mathrm{~N}$ isotope dilution technique was used to determine the biological nitrogen fixation of $P$. polymyxa WLY78 and $\triangle$ nifB- $V$ mutant. Cucumber plants derived $25.93 \pm$ $2.32 \%$ from the nitrogen fixation of $P$. polymyxa WLY78 under low nitrogen fixation, but the $\triangle$ nifB- $V$ mutant hardly fixed nitrogen. Similar reports are found that nitrogen fixation of $P$. beijingensis BJ-18 provided $27.8 \%$ nitrogen for cucumber under low nitrogen conditions (Li et al. 2019) and P. polymyxa P2b$2 \mathrm{R}$ provided $15 \%$ nitrogen for maize (Padda et al. 2017).

\section{Declarations}

Funding This work was supported by the National Key Research and Development Program of China (No. 2019YFA0904700).

Conflict of interest The authors have no conflicts of interest to declare.

Availability of data and material All data generated or analysed during this study are included in this published article.

Authors' contributions Conceptualization: Chen SF; Experiments: Liu S and Li Q; Methodology: Liu S, Li Q, Li YB and Hao TY; Writing: Liu S, Li Q and Chen SF; Funding acquisition: Chen SF. All authors read and approved the final manuscript.

\section{Ethics approval}

Not applicable

\section{References}

1. Amir HG., Shamsuddin ZH., Halimi MS., Ramlan MF, Marziah M (2003) $\mathrm{N}_{2}$ fixation, nutrient accumulation and plant growth promotion by rhizobacteria in association with oil palm seedlings. Pak J Biol Sci 6, 1269-1272. 
2. Baldani JI, Caruso L, Baldani VLD, Goi SR, Dobereiner J (1997) Recent advances in BNF with nonlegume plants. Soil Biol Biochem 29: 911-922. https://doi.org/10.1016/s0038-0717(96)00218-0.

3. Boddey RM, Deoliveira OC, Urquiaga S, Reis VM, Deolivares FL, Baldani VLD, Dobereiner J (1995) Biological nitrogen fixation associated with sugar cane and rice: contributions and prospects for improvement. Plant Soil 174: 195-209. https://doi.org/10.1007/bf00032247.

4. Chalk PM (1991) The contribution of associative and symbiotic nitrogen fixation to the nitrogen nutrition of non-legumes. Plant Soil 132: 29-39. https://doi.org/10.1007/bf00011009.

5. Dart PJ (1986) Nitrogen fixation associated with non-legumes in agriculture. Plant Soil 90: 303-334. https://doi.org/10.1007/bf02277405.

6. Farrar K, Bryant D, Cope-Selby N (2014) Understanding and engineering beneficial plant-microbe interactions: plant growth promotion in energy crops. Plant Biotechnol J 12: 1193-1206. https://doi.org/10.1111/pbi.12279.

7. Fox AR, Soto G, Valverde C, Russo D, Lagares A, Zorreguieta A, Alleva K, Pascuan C, Frare R, MercadoBlanco J, Dixon R, Ayub ND (2016) Major cereal crops benefit from biological nitrogen fixation when inoculated with the nitrogen-fixing bacterium Pseudomonas protegens Pf-5 X940. Environ Microbiol 18: 3522-3534. https://doi.org/10.1111/1462-2920.13376.

8. Geddes BA, Ryu MH, Mus F, Costas AG, Peters JW, Voigt CA, Poole P (2015) Use of plant colonizing bacteria as chassis for transfer of $\mathrm{N}_{2}$-fixation to cereals. Curr Opin Biotechnol 32: 216-222. https://doi.org/10.1016/j.copbio.2015.01.004.

9. Hao TY, Chen SF (2017) Colonization of wheat, maize and cucumber by Paenibacillus polymyxa WLY78. PLoS One 12: e0169980. https://doi.org/10.1371/journal.pone.0169980.

10. Hurek T, Handley LL, Reinhold-Hurek B, Piche Y (2002) Azoarcus grass endophytes contribute fixed nitrogen to the plant in an unculturable state. Mol Plant Microbe In 15: 233-242. https://doi.org/10.1094/mpmi.2002.15.3.233.

11. Iniguez AL, Dong YM, Triplett EW (2004) Nitrogen fixation in wheat provided by Klebsiella pneumoniae 342. Mol Plant Microbe In 17: 1078-1085. https://doi.org/10.1094/mpmi.2004.17.10.1078.

12. Ke XB, Feng S, Wang J, Lu W, Zhang W, Chen M, Lin M (2019) Effect of inoculation with nitrogenfixing bacterium Pseudomonas stutzeri A1501 on maize plant growth and the microbiome indigenous to the rhizosphere. Syst Appl Microbiol 42: 248-260. https://doi.org/10.1016/j.syapm.2018.10.010.

13. Li YB, Li YL, Zhang HW, Wang MY, Chen SF (2019) Diazotrophic Paenibacillus beijingensis BJ-18 provides nitrogen for plant and promotes plant growth, nitrogen uptake and metabolism. Front Microbiol 10: 1119. https://doi.org/10.3389/fmicb.2019.01119.

14. Li YL, Chen SS (2019) Fusaricidin produced by Paenibacillus polymyxa WLY78 induces systemic resistance against Fusarium wilt of cucumber. Int J Mol Sci 20: 5240. https://doi.org/10.3390/ijms20205240. 
15. Oliveira ALM, Santos OJAP, Marcelino PRF, Milani KML, Zuluaga MYA, Zucareli C, Goncalves LSA (2017) Maize inoculation with Azospirillum brasilense Ab-V5 cells enriched with exopolysaccharides and polyhydroxybutyrate results in high productivity under low $\mathrm{N}$ fertilizer input. Front Microbiol 8 : 1873. https://doi.org/10.3389/fmicb.2017.01873.

16. Padda KP, Puri A, Zeng QW, Chanway CP, Wu XQ (2017) Effect of GFP-tagging on nitrogen fixation and plant growth promotion of an endophytic diazotrophic strain of Paenibacillus polymyxa. Botany 95: 933-942. https://doi.org/10.1139/cjb-2017-0056.

17. Raymond J, Siefert JL, Staples CR, Blankenship RE (2004) The natural history of nitrogen fixation. Mol Biol Evol 21: 541-554. https://doi.org/10.1093/molbev/msh047.

18. Setten L, Soto G, Mozzicafreddo M, Fox AR, Lisi C, Cuccioloni M, Angeletti M, Pagano E, Diaz-Paleo A, Ayub ND (2013) Engineering Pseudomonas protegens Pf-5 for nitrogen fixation and its application to improve plant growth under nitrogen-deficient conditions. PLoS One 8: e63666. https://doi.org/10.1371/journal.pone.0063666.

19. Sevilla M, Burris RH, Gunapala N, Kennedy C (2001) Comparison of benefit to sugarcane plant growth and ${ }^{15} \mathrm{~N}_{2}$ incorporation following inoculation of sterile plants with Acetobacter diazotrophicus wild-type and $\mathrm{Nif}^{-}$mutant strains. Mol Plant Microbe In 14: 358-366. https://doi.org/10.1094/mpmi.2001.14.3.358.

20. Van Deynze A, Zamora P, Delaux PM, Heitmann C, Jayaraman D, Rajasekar S, Graham D, Maeda J, Gibson D, Schwartz KD, Berry AM, Bhatnagar S, Jospin G, Darling A, Jeannotte R, Lopez J, Weimer BC, Eisen JA, Shapiro HY, Ane JM, Bennett AB (2018) Nitrogen fixation in a landrace of maize is supported by a mucilage-associated diazotrophic microbiota. PLoS Biol 16: e2006352. https://doi.org/10.1371/journal.pbio.2006352.

21. Wang LY, Zhang LH, Liu ZZ, Zhao DH, Liu XM, Zhang B, Xie JB, Hong YY, Li PF, Chen SF, Dixon R, Li $\mathrm{JL}$ (2013) A minimal nitrogen fixation gene cluster from Paenibacillus sp. WLY78 enables expression of active nitrogenase in Escherichia coli. PLoS Genet 9: e1003865. https://doi.org/10.1371/journal.pgen.1003865.

22. Wang TS, Zhao XY, Shi HW, Sun L, Li YB, Li Q, Zhang HW, Chen SF, Li JL (2018) Positive and negative regulation of transferred nif genes mediated by indigenous GInR in Gram-positive Paenibacillus polymyxa. PLoS Genet 14: e1007629. https://doi.org/10.1371/journal.pgen.1007629.

23. Xie JB, Du ZL, Bai LQ, Tian CF, Zhang YZ, Xie JY, Wang TS, Liu XM, Chen X, Cheng Q, Chen SF, Li JL (2014) Comparative genomic analysis of $\mathrm{N}_{2}$-fixing and non- $\mathrm{N}_{2}$-fixing Paenibacillus spp.: organization, evolution and expression of the nitrogen fixation genes. PLoS Genet 10: e1004231. https://doi.org/10.1371/journal.pgen.1004231.

24. Xie JB, Shi HW, Du ZL, Wang TS, Liu XM, Chen SF (2016) Comparative genomic and functional analysis reveal conservation of plant growth promoting traits in Paenibacillus polymyxa and its closely related species. Sci Rep 6: 21329. https://doi.org/10.1038/srep21329.

25. Xu PX, Han LL, He JZ, Luo F, Zhang LM (2017) Research advance on molecular ecology of asymbiotic nitrogen fixation microbes. J Appl Ecol 28: 3440-3450. doi: 10.13287/j.1001- 


\section{Figures}

a

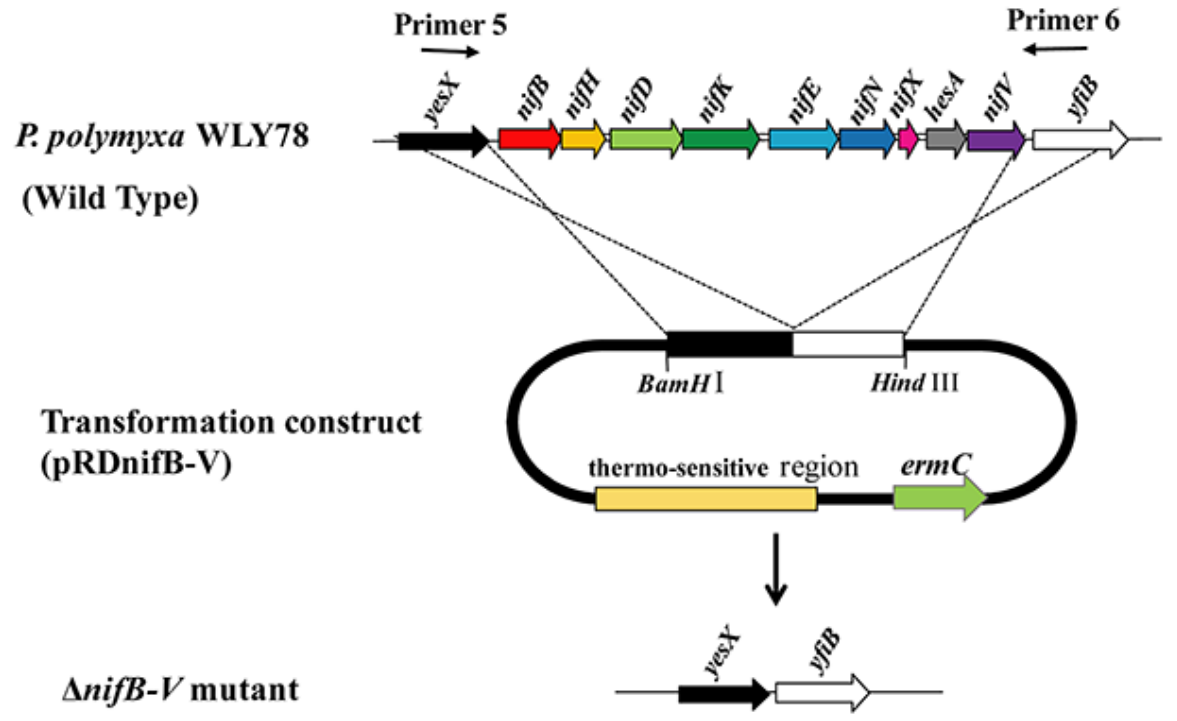

b

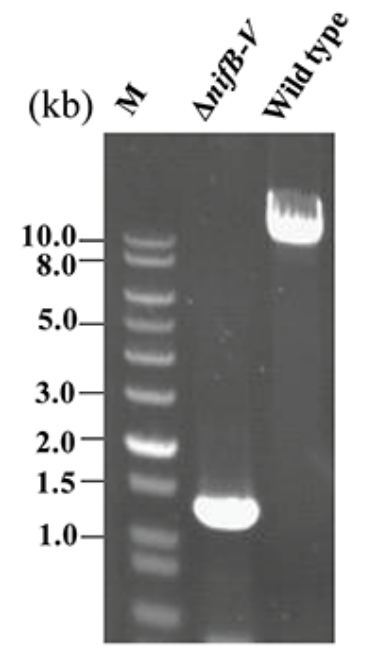

$\mathrm{C}$

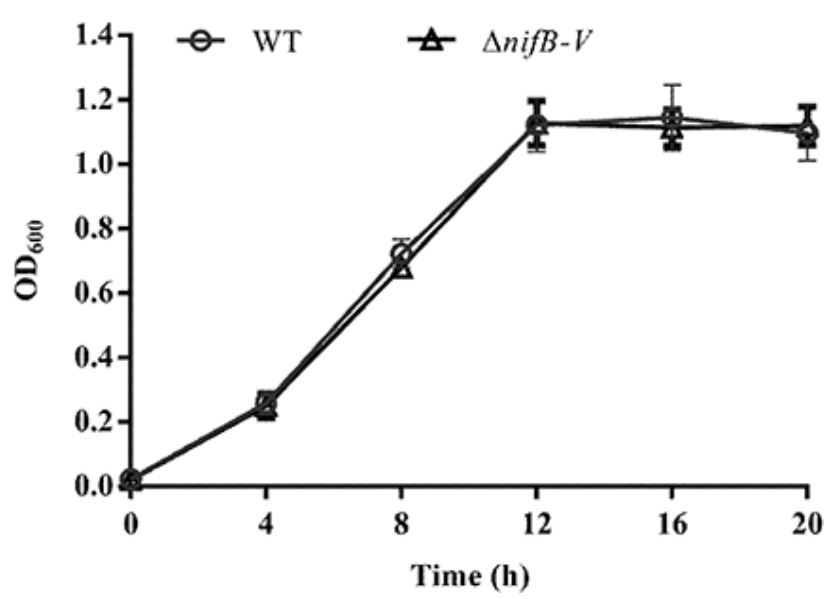

d

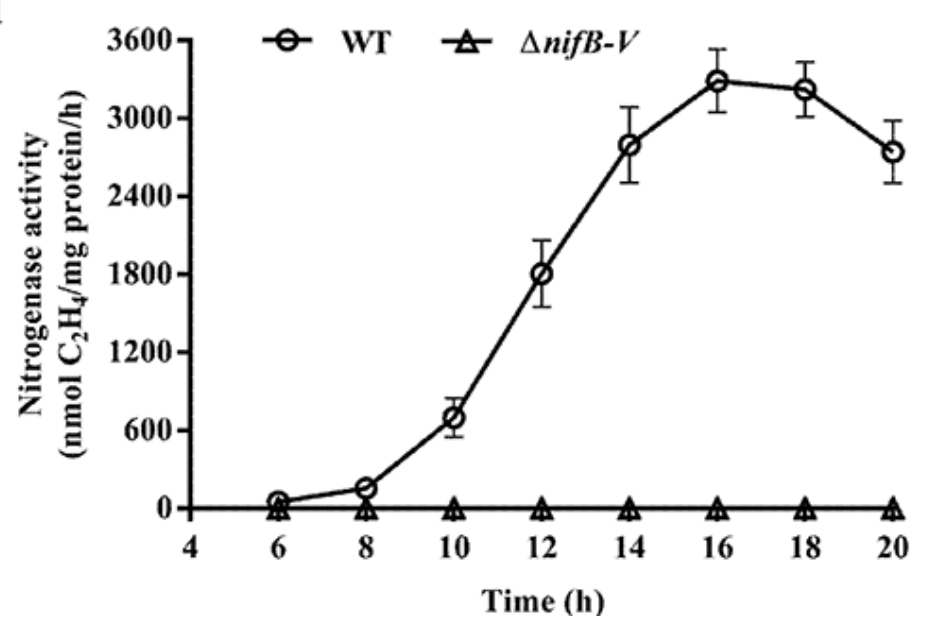

Figure 1

Construction and characterization of nif gene cluster deletion mutant ( $\triangle$ nifB-V) of P. polymyxa WLY78. a. General scheme of construction of nif gene cluster deletion mutant. b. The nif cluster disruption was confirmed by PCR using the primer 5 and primer 6. c. Comparison of growth of the WT (the wild-type) and $\Delta$ nifB-V (deletion mutant) strains. Growth of the WT and $\Delta$ nifB-V strains in nitrogen-limited medium supplemented with $100 \mathrm{mM} \mathrm{NH} 4 \mathrm{Cl}$ as the nitrogen source. $\mathrm{d}$. Nitrogenase activities of WT and $\Delta$ nifB-V grown anaerobically in nitrogen-limited medium. 
a

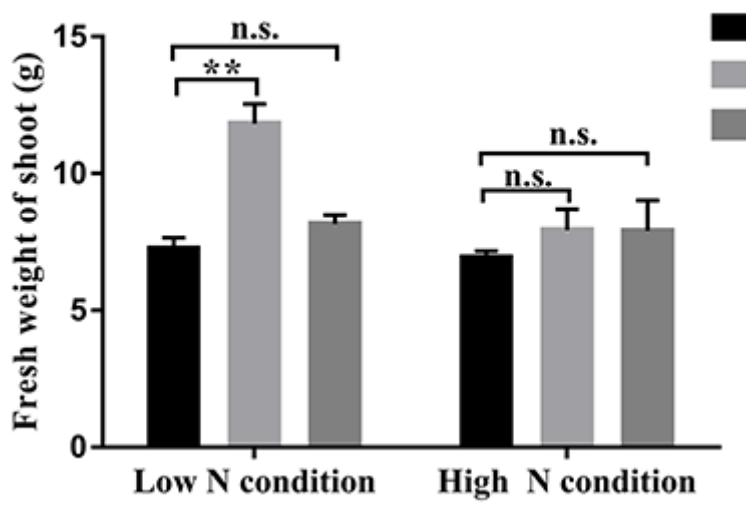

C

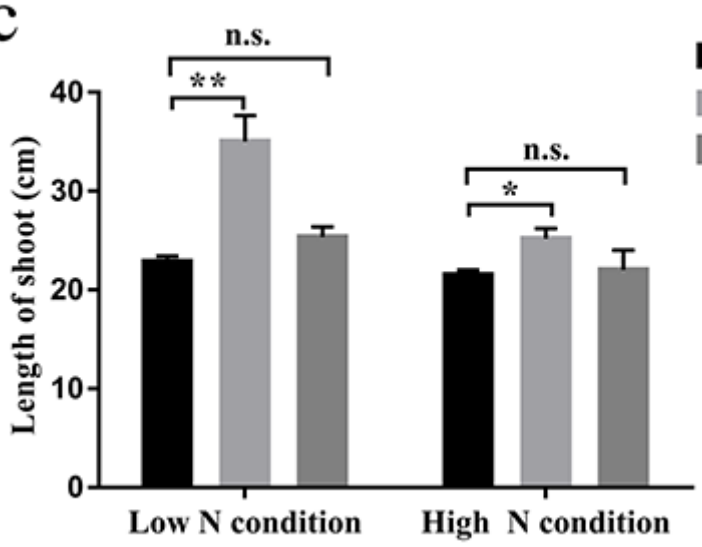

Control

WT

$\Delta n i f B-V$ b

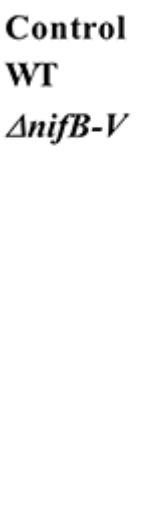

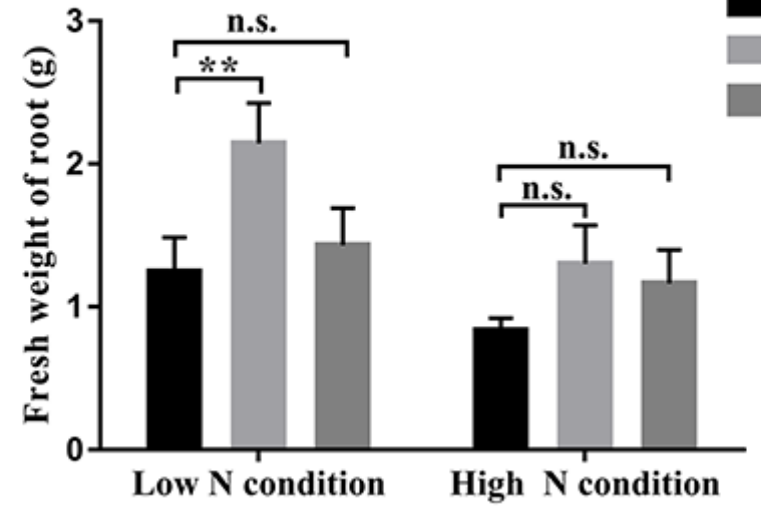

d

Control

WT

$\Delta n i f B-V$

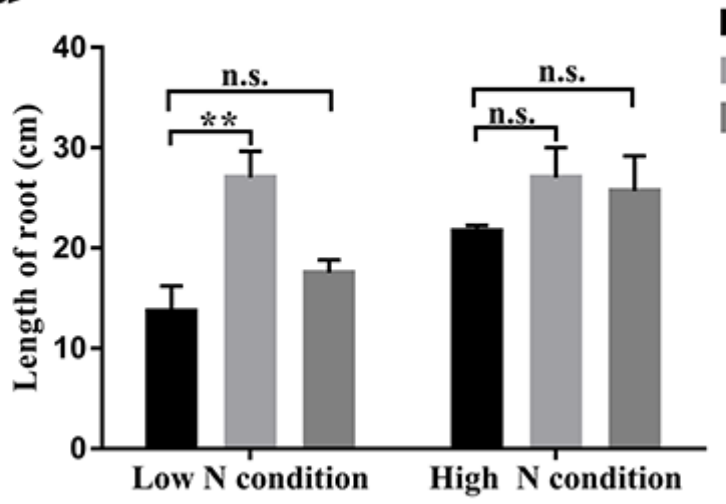

Control

WT

$\Delta n i f B-V$

Figure 2

Colonization of GFP-labeled P. polymyxa WLY78 and GFP-labeled $\triangle$ nifB-V mutant in cucumber.

Colonization pattern of P. polymyxa WLY78 in root (a), stem (b) and leaf (c). Colonization pattern of $\Delta$ nifB-V mutant in root (d), stem (e) and leaf (f). Scale bars $(a-f)=100 \mu \mathrm{m}$. 
a

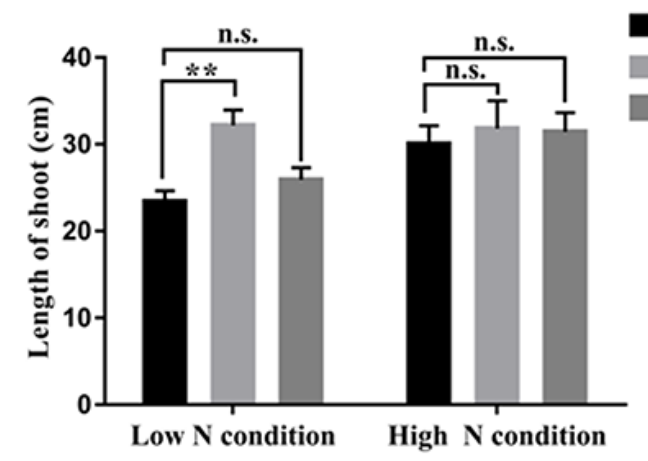

c

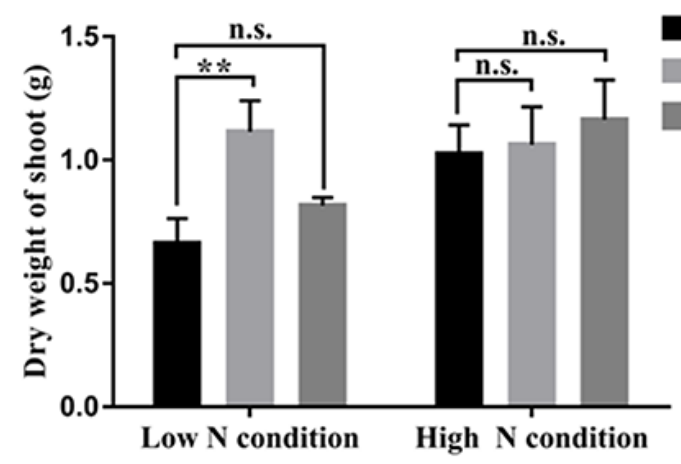

b

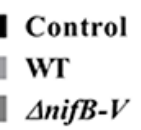

Control

WT

$\Delta n i f B-V$

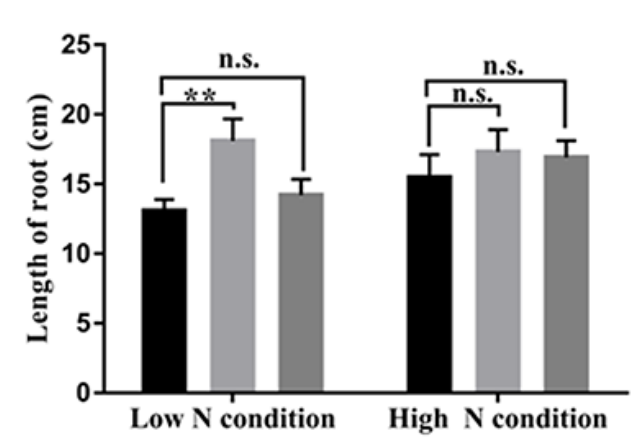

d

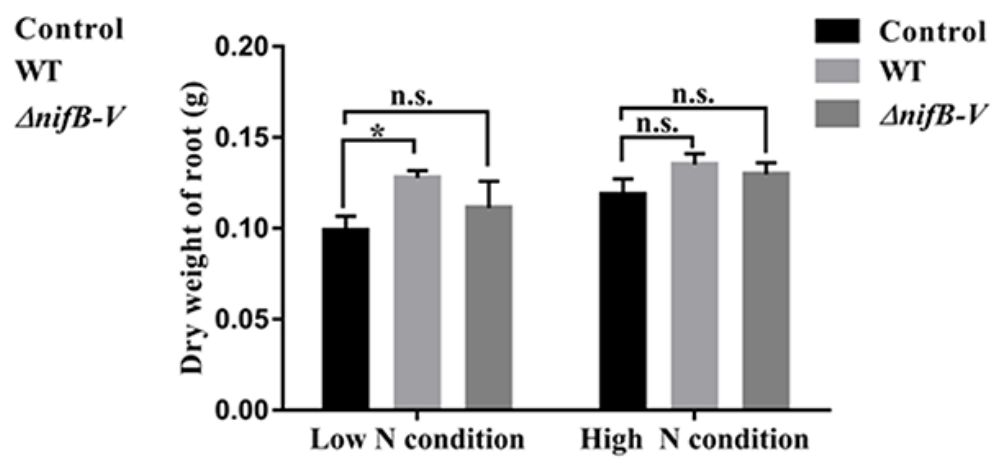

\section{Figure 3}

Biomass of cucumber inoculated with P. polymyxa WLY78 and $\triangle$ nifB-V mutant, with non-inoculated cucumber as control. Length of shoots (a) and roots (b). Dry weights of shoots (c) and roots (d). The error bars in the figure indicate SD values. Statistical analysis is performed using One-way ANOVA. * Indicates $P<0.05$, ** indicates $P<0.01$. 

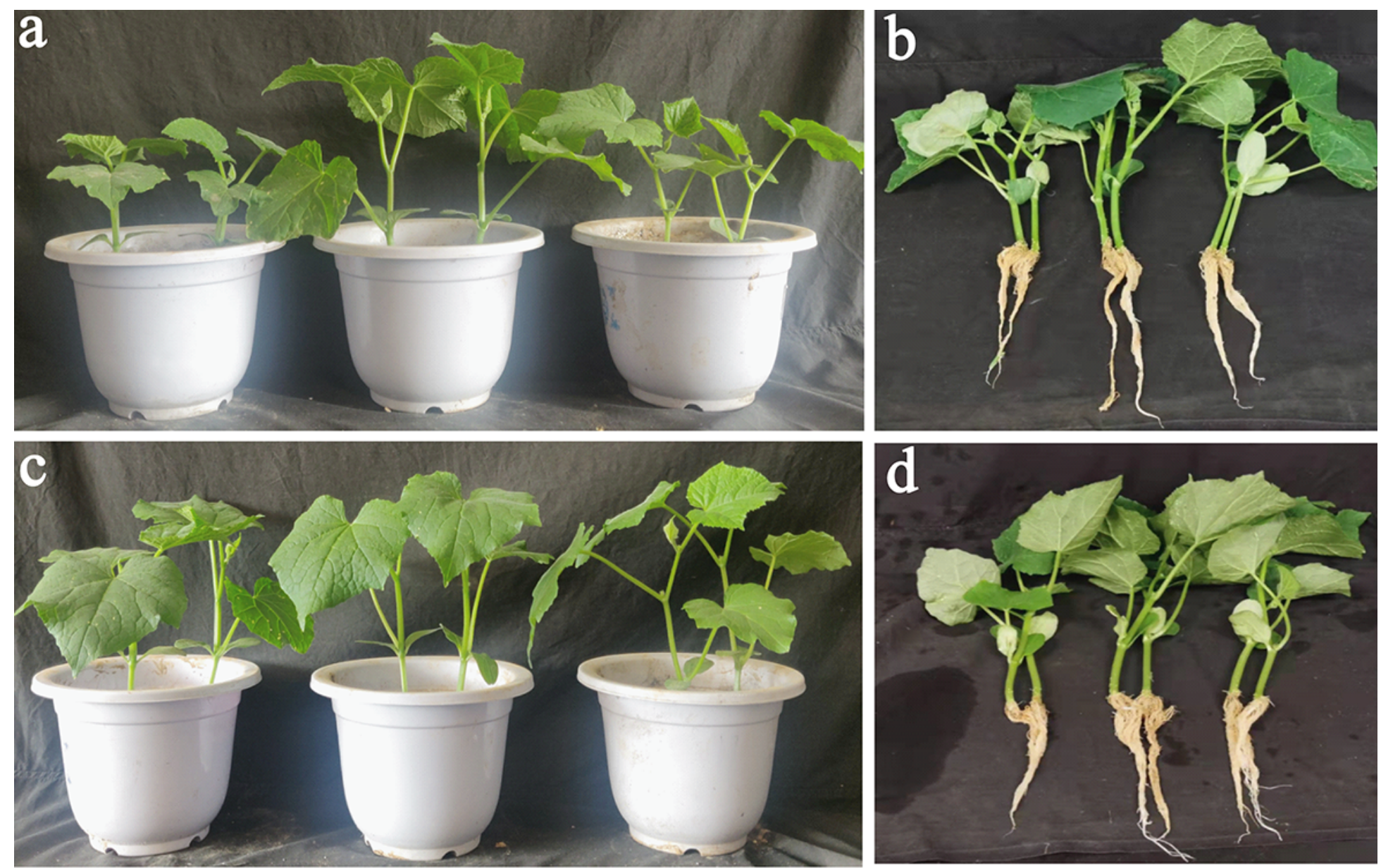

CK

WT

$\Delta n i f B-V$

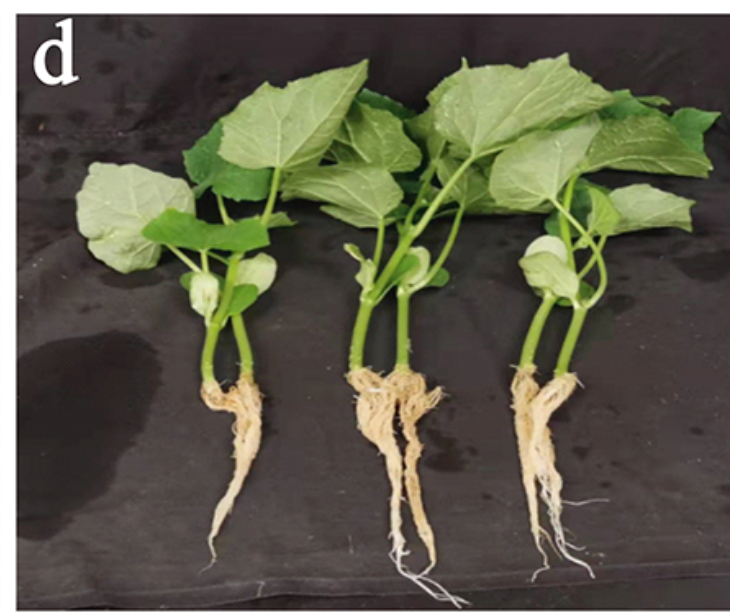

CK WT $\Delta$ nifB- $V$

\section{Figure 4}

Cucumber cultivation in greenhouse with P. polymyxa WLY78 and $\triangle$ nifB-V mutant, with non-inoculated cucumber as control. The growth of cucumber seedlings under low (a) and high (c) nitrogen conditions. The shoot and root of cucumber under low (b) and high (d) nitrogen conditions. 